\title{
Skin Microvascular Thrombosis in Fusarium Infection in Two Early Biopsied Cases
}

\author{
Yang Fan ${ }^{a, b}$ Lise Willems ${ }^{c}$ Christophe Leboeuf ${ }^{a, b}$ \\ Wang Li ${ }^{a, b} \quad$ Claire Lacroix ${ }^{b, d}$ Marie Robin $^{e}$ \\ Gérard Sociéa, b, e Patricia Ribaud $^{b, e} \quad$ Laurence Verneuil ${ }^{a, f}$ \\ Anne Janin ${ }^{a, b, c}$ \\ anserm, U728, bUniversité Paris 7, 'AP-HP, Hôpital Saint-Louis, Service de \\ Pathologie, 'AP-HP, Hôpital Saint-Louis, Service de Mycologie, and 'Service des \\ Greffes, Paris; ${ }^{\mathrm{f}} \mathrm{CHU}$ Caen, Service de Dermatologie, Caen, France
}

\section{Key Words}

Skin biopsy · Microvessel · Thrombosis · Fusarium

\begin{abstract}
Fusarium species cause rare and severe infections. Their incidence is increasing in immunocompromised patients but they are also observed in healthy hosts. Because of the rapid dissemination of infection and the frequent resistance of Fusarium species to antifungal drugs, histopathologic evidence of hyphae is very helpful to obtain the diagnosis rapidly. We report the clinical and pathological features of two patients with initial cutaneous lesions. Cutaneous early biopsies showed microvessel involvement with hyphae and thrombosis. Fusarium infection was confirmed by skin culture. Hyphae within a microvessel thrombus in the skin were highly suggestive of disseminated fungal infection. These pathological features enabled to establish an early diagnosis and to start efficient antifungal treatment. In early cutaneous biopsies of immunocompromised patients, the presence of cutaneous vessel thrombosis can suggest a fungal infection and may help to start specific therapy without delay for these life-threatening infections.
\end{abstract}

\section{Introduction}

Fusarium species cause a broad spectrum of human diseases, ranging from superficial or localized infections such as corneal infections, endophthalmitis or onychomycosis in healthy hosts, to severe disseminated infection in immunocompromised patients. 23-30\% of documented fusariosis cases occur after haematopoietic stem cell transplantation (HSCT) $[1,2]$ and, in this population, Fusarium is the second cause of mold infections 
after Aspergillus [3]. These infections are associated with high mortality rates (70-87\%) and their prognosis remains poor [4]. Disseminated infections often develop from an initial skin infection. Fusarium species possess angiotropic and angioinvasive properties resulting in thrombosis and tissue necrosis. The diagnosis of Fusarium infection is determined by blood and/or tissue biopsy culture. The latter is of great importance because blood culture may be negative despite blood dissemination.

We report two cases of disseminated fusariosis with cutaneous lesions, whose biopsies, showing hyphae within a microvessel thrombus, enabled to establish an early diagnosis.

\title{
Cases Reports
}

\author{
Case 1 (fig. 1a)
}

A 16-year-old man with hyperleukocytic undifferentiated acute leukaemia secondary to Fanconi anaemia underwent stem cell transplantation with double mismatched unrelated umbilical cord blood (UCB). Three months before this procedure he had received two courses of chemotherapy with FLAG regimen (fludarabine, aracytine and granulocyte-colony-stimulating factor) which had reduced the medullary blasts count. However, he kept a severe pancytopenia until his admission for double UCB transplant, realized after a nonmyeloablative conditioning regimen with total body irradiation, fludarabine and cyclophosphamide. Graft versus host disease prophylaxis was methylprednisolone $(1 \mathrm{mg} / \mathrm{kg})$, cyclosporine and mycophenolate mofetil. The patient received a second mismatched unrelated UCB transplant 7 weeks after the first transplant. One day before the second transplant, he developed violaceous, indurated skin lesions and purpuric blisters on the wrist, with fever and elevated C-reactive protein despite broad-spectrum antibiotherapy and caspofungin. Skin biopsy, performed two days after the onset of the lesions, showed septate, acute-branching hyphae and macroconidia within a microvessel thrombus. Antifungal biotherapy associating voriconazole and liposomal amphotericin B was immediately started. Progression of skin lesions on both arms, suggestive of disseminated infection despite negative blood culture, required the increase of liposomal amphotericin B. Cerebral and lung imagery and coagulation tests were normal. No skin necrosis occurred and the lesions progressively resolved to disappear by day 45 . The patient later developed candidemia and acute graft versus host disease with digestive haemorrhage and died 69 days after the second graft.

\section{Case 2 (fig. 1b)}

A 50-year-old woman, who had had a renal transplant in 2000, followed by $10 \mathrm{mg}$ prednisone and $1.5 \mathrm{~g}$ mycophenolate mofetil daily, developed painful erythematous papules on the leg, with fever for 3 days. C-reactive protein was elevated. Physical examination showed erythematous papules centered by pustules with a homolateral inguinal adenopathy. Lung scan was normal. Skin biopsy, performed 3 days after the onset of the eruption, showed broad, septate hyphae with branching ankles and macroconidia within a microvessel thrombus. Blood culture was negative. Antifungal biotherapy, associating voriconazole and liposomal amphotericin B, was immediately started. No skin necrosis occurred and skin lesions disappeared by day 40 .

\section{Pathological Findings}

Skin biopsies were performed early in the course of the disease (two and three days after the onset of eruption), before any treatment. They allowed the epidermis, full dermis and subcutaneous fat analyses. The lesions were found in the papillary dermis (fig. 1a) and in the hypodermis (fig. 1b) in the microvessel network. In the two cases, microvessel thrombus was associated with septate, acute-branching hyphae and macroconidia well shown on special stain. Skin cultures isolated Fusarium sp. Fusarium subglutinans, close to Gibberella fujikuroi complex was identified by DNA sequencing in case 1 (Centre National de Référence des Mycoses et des Antifongiques, Institut Pasteur, Paris, France). Fusarium solani was identified by phenotypic methods in case 2 . The cultures yielded colonies that were white to creamy in colour with a loccose aerial mycelium. Slide cultures on Sabouraud's agar revealed oval microconidia sometimes grouped in verticilliums produced from elongated lateral phialides. We observed a few intercalary, rough-walled chlamydospores [5]. 


\begin{tabular}{c|l|l|l} 
Case Reports $h$ & Case Rep Dermatol 2010;2:76-81 & Published online: May 7, 2010 & $\begin{array}{l}\text { @ 2010 S. Karger AG, Basel } \\
\text { ISSN 1662-6567 } \\
\text { www.karger.com/cde }\end{array}$ \\
\hline Dol: $10.1159 / 000313934$ & & & \\
\hline
\end{tabular}

\section{Discussion}

Skin biopsy early in the course of two cases of Fusarium infection allowed us to detect microvessel thrombosis and hyphae in the papillary dermis and hypodermis. Fusarium infection incidence is growing in immunocompromised patients and reported rates vary from 0.4 to $2 \%$ after allogeneic HSCT [6]. However, these infections can also occur in healthy persons, resulting in keratitis or onychomycosis or less frequently in skin infection after face trauma, eye surgery, burns or wounds [2]. For immunocompromised patients, profound and prolonged neutropenia and high doses of corticosteroids are the most important underlying risk factors. Other risk factors include HSCT, HLAmismatched compared to HLA-matched transplant and central venous catheter use $[1,4]$. Persistent neutropenia is also a major predictor of mortality [4]. Our two cases were immunocompromised. The onset of the fusariosis infection was characterized by skin lesions.

Skin has been found to be involved in more than $70 \%$ of patients with Fusarium infection and this incidence increases until $75-90 \%$ in disseminated infections [2]. Skin infectious entry door concerned $33 \%$ of affected patients [1]. The disease can be limited to the initial skin lesion, or extend to other skin locations or other organs, predominantly blood (70\%), lungs (39\%) and sinuses (18\%) [3]. Even though, in these cases, blood culture was negative, detection of Fusarium inside the thrombosis suggested that blood was probably involved. Usually, skin lesions are macules or papules with or without central necrosis, pustules, or subcutaneous nodules [7]. Bodey et al. have observed necrosis of skin papules in more than $60 \%$ of disseminated Fusarium infections [2]. Haemorrhage in internal organs has also been described after autopsy [8]. This has led to the hypothesis that skin damage in Fusarium infection involves the vessels, and that thrombosis explains the frequent necrotic feature. In our cases, early skin biopsies allowed us to find microvessel thrombosis, associated with hyphae, but we did not observe necrosis. In an animal study, necrotizing abscesses with acute branching septate hyphae, neutrophil and macrophage infiltration were found in nonneutropenic mice, whereas neutropenic mice did not have inflammation and had a higher fungal burden [8]. The absence of necrosis in our two patients could be linked to the fact that they were neutropenic, and/or to the fact that they were biopsied early, before necrosis followed microvessel thrombosis.

Among the 8 cases of fusariosis with hyphae presence within skin vessels reported in the literature (table 1), only two cases showed thrombosis in large vessels (retinal vessel and superficial suppurative thrombophlebitis after antecubital intravenous catheter). However, 7 out of the 8 cases had skin necrosis, which suggests that microvessel thrombosis is a frequent event. In our cases, the fact that the skin biopsies were performed early in the course of the disease allowed us to recognize microvessel thrombosis with hyphae. Rapid start of the specific treatment might have prevented worsening of skin lesions and further necrosis.

Early skin biopsy also allows the fungal culture from the skin specimen. The diagnosis of Fusarium infection was established on skin sample culture in our cases. The value of skin sample culture has been recently underlined, since the blood culture isolates the Fusarium in only approximately $70 \%$ of reported cases [2]. However, cultures require several days to enable a diagnosis whereas histology results can be obtained more rapidly. Specific stains show hyphae filaments (phialides) and unicellular form spores (phialoconidia) in a closed lesion [8]. However, discrimination between Fusarium species 
and other non-Aspergillus hyalohyphomycoses species is difficult by histopathologic examination and culture remains the standard for identification of these fungi.

Differential diagnosis with Aspergillus can be difficult. Both Aspergillus and Fusarium progress through vascular invasion with sometimes vascular wall rupture leading to severe tissue damage. Histopathology shows similar acute branching septate hyphae although hyphae branching of both 45 and 90 degrees are present and hyphae diameter is smaller and irregular in Fusarium in opposite to Aspergillus. Furthermore, immunostainings with specific monoclonal antibodies not performed in our cases have proved to be a powerful tool for the accurate diagnosis of systemic mycoses, including aspergillosis and fusariosis [9].

In the treatment of invasive fungal infections, systematic antifungal susceptibility testing is recommended [6]. Because of a lack of clinical trials and the critical role of immune reconstitution in the outcome of fusariosis, the optimal treatment strategy for patients with severe fusarial infection has to be defined. In a retrospective analysis of 84 patients with haematologic diseases and invasive fusariosis, treatment was deoxycholate amphotericin or a lipid formulation of amphotericin B. 27 patients (32\%) responded to treatment, but only 18 patients $(21 \%)$ were still alive 90 days after diagnosis. The response rate to a lipid formulation of amphotericin $B$ appeared to be superior to that to deoxycholate amphotericin B ( $46 \%$ vs. $32 \%$, respectively), but the difference was not statistically significant $(\mathrm{p}<0.36)[10]$. In another study, voriconazole was given to 11 patients with fusariosis, all intolerant or refractory to primary therapy. The response rate was $45 \%$, with a 90 -day actuarial survival of $71 \%$ [11]. Posaconazole has also been used as salvage therapy among 21 patients with proven or probable fusariosis. All but one patient were initially treated with a lipid-based formulation of amphotericin B. The overall success rate (complete plus partial response) was 48\% [12]. Data on combination therapy for fusariosis are limited to a few case reports: caspofungin plus amphotericin $B$ [13], amphotericin B plus voriconazole [14], amphotericin B and terbinafine [15]. Given the scarcity of data, no solid recommendations for combination therapy can be provided. The prevention and treatment of invasive fungal infections, aspergillosis and fusariosis, include standard and liposomal formulations of amphotericin B. Newer azoles, particularly voriconazole and posaconazole, have an enhanced spectrum of activity including Aspergillus and Fusarium species. Recently, therapeutic recommendations in invasive fusariosis suggest a high dose of amphotericin B for F. solani/F. verticolloides and a high dose of amphotericin B or voriconazole for other Fusarium species [6].

To conclude, in early cutaneous biopsies of immunocompromised patients, the presence of cutaneous vessel thrombosis can suggest a fungal infection and presence of hyphae confirms the fungal infection. Vessel thrombosis may help to start specific therapy without delay for these life-threatening infections. 


\begin{tabular}{c|l|l|l}
$\begin{array}{c}\text { Case Reports } h \\
\text { Denmofology }\end{array}$ & $\begin{array}{l}\text { Case Rep Dermatol 2010;2:76-81 } \\
\text { D01: } 10.1159 / 000313934\end{array}$ & Published online: May 7, 2010 & $\begin{array}{l}\text { O 2010 S. Karger AG, Basel } \\
\text { ISSN 1662-6567 } \\
\text { www.karger.com/cde }\end{array}$ \\
\hline
\end{tabular}

Table 1. Reported cases of Fusarium infections with hyphae presence within skin vessels

\begin{tabular}{|c|c|c|c|c|c|c|c|c|c|}
\hline Case & Age & Sex & Species & Outcome & Initial disease & Type of infection & $\begin{array}{l}\text { Skin } \\
\text { necrosis }\end{array}$ & $\begin{array}{l}\text { Throm- } \\
\text { bosis }\end{array}$ & $\begin{array}{l}\text { Refer- } \\
\text { ence }\end{array}$ \\
\hline 1 & 20 & $\mathrm{~F}$ & oxysporum & died & $\mathrm{ALL}+\mathrm{BMT}$ & disseminated & yes & no & 7 \\
\hline 2 & 59 & M & oxysporum & died & $\mathrm{ALL}+\mathrm{BMT}$ & disseminated & yes & no & 8 \\
\hline 3 & 29 & M & NA & unknown & ALL & disseminated & yes & no & 9 \\
\hline 4 & 53 & M & solani & died & renal graft & disseminated & yes & no & 10 \\
\hline 5 & 51 & $\mathrm{M}$ & NA & died & AIDS & disseminated & yes & yes & 11 \\
\hline 6 & 35 & $\mathrm{~F}$ & moniliforme & died & ALL & skin only & yes & no & 12 \\
\hline 7 & 5 & $\mathrm{M}$ & moniliforme & died & ALL & skin only & yes & no & 13 \\
\hline 8 & 79 & $\mathrm{~F}$ & proliferatum & resolved & face trauma & vein & no & yes & 14 \\
\hline $9^{*}$ & 16 & $\mathrm{M}$ & subglutinans & died & HUAL & skin & no & yes & \\
\hline $10^{*}$ & 50 & $\mathrm{~F}$ & solani & resolved & renal graft & skin & no & yes & \\
\hline
\end{tabular}

$\mathrm{F}=$ Female; $\mathrm{M}=$ male; $\mathrm{NA}=$ not available; $\mathrm{ALL}=$ acute lymphoid leukaemia; $\mathrm{BMT}=$ bone marrow transplantation; AIDS = acquired immunodeficiency syndrome; HUAL = hyperleukocytic undifferentiated acute leukaemia. ${ }^{*}$ Present study.

Fig. 1. a Case 1: Skin biopsy performed two days after the eruption onset. Left: purpuric blisters; middle: microvessel thrombosis in the papillary dermis, hematoxylin-eosin stain, $\times 400$; right: following section at the same magnification; presence of multiple hyphae within the microvessel thrombus, Gomori-Grocott stain (scale bar $=300 \mu \mathrm{m})$. b Case 2: Skin biopsy performed three days after the eruption onset. Left: erythematous papules centered by pustules; middle: microvessel thrombosis in the hypodermis, hematoxylin-eosin stain, $\times 400$; right: reticulin silver stain showing the basal lamina of the microvessel and hyphae within the thrombus, following section, $\times 400$ (scale bar $=300 \mu \mathrm{m})$.

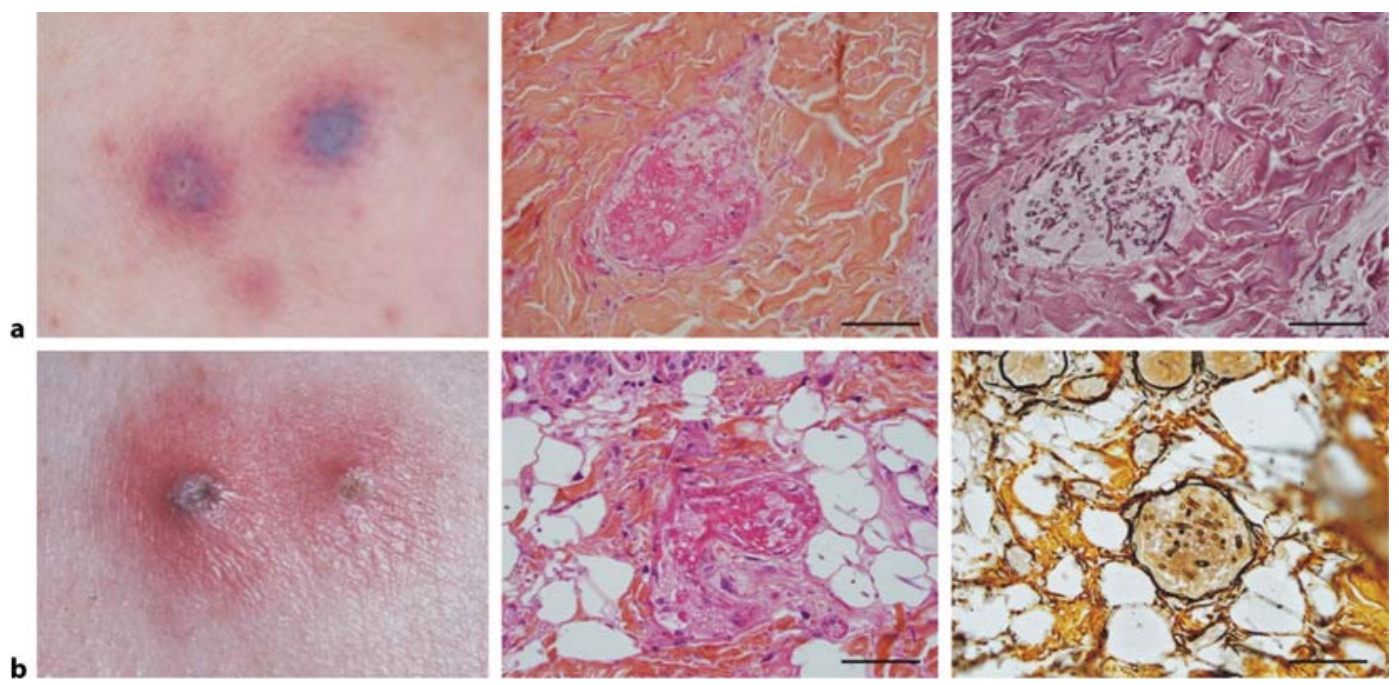




\section{References}

1 Boutati EI, Anaissie EJ: Fusarium, a significant emerging pathogen in patients with hematologic malignancy: ten years' experience at a cancer center and implications for management. Blood 1997;90:999-1008.

-2 Bodey GP, Boktour M, Mays S, Duvic M, Kontoyiannis D, Hachem R, Raad I: Skin lesions associated with Fusarium infection. J Am Acad Dermatol 2002;47:659-666

-3 Nucci M, Marr KA, Queiroz-Telles F, Martins CA, Trabasso P, Costa S, Voltarelli JC, Colombo AL, Imhof A, Pasquini R, Maiolino A, Souza CA, Anaissie E: Fusarium infection in hematopoietic stem cell transplant recipients. Clin Infect Dis 2004;38:1237-1242.

4 Cornely OA: Aspergillus to Zygomycetes: causes, risk factors, prevention, and treatment of invasive fungal infections. Infection 2008;36:296-313.

5 De Hoog GS, Guarro J, Gené J, Figueras MJ: Atlas of Clinical Fungi. Utrecht, ASM Press, 2000.

6 Nucci M, Anaissie E: Fusarium infections in immunocompromised patients. Clin Microbiol Rev 2007;20:695-704.

7 Acland KM, Hay RJ, Groves R: Cutaneous infection with Alternaria alternata complicating immunosuppression: successful treatment with itraconazole. Br J Dermatol 1998;138:354-356.

-8 Saito T, Imaizumi M, Kudo K, Hotchi M, Chikaoka S, Yoshinari M, Suwabe N, Sato A, Suzuki H, Iinuma K: Disseminated Fusarium infection identified by the immunohistochemical staining in a patient with a refractory leukemia. Tohoku J Exp Med 1999;187:71-77.

-9 Jensen HE, Schonheyder HC, Hotchi M, Kaufman L: Diagnosis of systemic mycoses by specific immunohistochemical tests. APMIS 1996;104:241-258

10 Nucci M, Anaissie EJ, Queiroz-Telles F, Martins CA, Trabasso P, Solza C, Mangini C, Simoes BP, Colombo AL, Vaz J, Levy CE, Costa S, Moreira VA, Oliveira JS, Paraguay N, Duboc G, Voltarelli JC, Maiolino A, Pasquini R, Souza CA: Outcome predictors of 84 patients with hematologic malignancies and Fusarium infection. Cancer 2003;98:315-319.

-11 Perfect JR, Marr KA, Walsh TJ, Greenberg RN, DuPont B, de la Torre-Cisneros J, Just-Nubling G, Schlamm HT, Lutsar I, Espinel-Ingroff A, Johnson E: Voriconazole treatment for less-common, emerging, or refractory fungal infections. Clin Infect Dis 2003;36:1122-1131.

12 Raad II, Hachem RY, Herbrecht R, Graybill JR, Hare R, Corcoran G, Kontoyiannis DP: Posaconazole as salvage treatment for invasive fusariosis in patients with underlying hematologic malignancy and other conditions. Clin Infect Dis 2006;42:1398-1403.

13 Makowsky MJ, Warkentin DI, Savoie ML: Caspofungin and amphotericin B for disseminated Fusarium verticillioides in leukemia. Ann Pharmacother 2005;39:1365-1366.

14 Durand-Joly I, Alfandari S, Benchikh Z, Rodrigue M, Espinel-Ingroff A, Catteau B, Cordevant C, Camus D, Dei-Cas E, Bauters F, Delhaes L, De Botton S: Successful outcome of disseminated Fusarium infection with skin localization treated with voriconazole and amphotericin B-lipid complex in a patient with acute leukemia. J Clin Microbiol 2003;41:4898-4900.

15 Rothe A, Seibold M, Hoppe T, Seifert H, Engert A, Caspar C, Karthaus M, Fatkenheuer G, Bethe U, Tintelnot K, Cornely OA: Combination therapy of disseminated Fusarium oxysporum infection with terbinafine and amphotericin B. Ann Hematol 2004;83:394-397.

Yang Fan and Lise Willems contributed equally. 